\title{
REFLEXIONES SOBRE LA GEOMETRÍA GRIEGA
}

\section{THOUGHTS ON THE GREEK GEOMETRY}

\author{
Piedad YusTE \\ Departamento de Filosofía \\ UNED
}

RESUMEN: La geometría griega incorpora una serie de elementos que carecen de consistencia en el mundo sensible, como son el punto, la línea y el plano, pero que surgen por la necesidad de superar la indefinición que la idea de infinito suscitaba en las mentes de los filósofos griegos. La introducción de estos elementos en la composición de cuadraturas originó una serie de paradojas y contradicciones que persistieron hasta el descubrimiento, en pleno siglo XVII, del cálculo integral. Aquí se revisan algunos conceptos esenciales de la geometría euclídea y se analizan brevemente los métodos de exhaución practicados por Hipócrates, Euclides y Arquímedes, observando cómo este último se encuentra más próximo a las tradiciones prácticas provenientes de Mesopotamia, mientras Euclides continúa fiel a la corriente platónico-aristotélica. griega.

DESCRIPTORES: Exhaución, Filosofía griega, Geometría

ABSTRACT: Greek geometry includes a number of elements with no reliance on the world of the senses, such as the point, the line and the plane. But they arise from the need to overcome the uncertainty that the idea of infinite raised in the minds of Greek philosophers. The introduction of these elements, in the composition of quadratures, caused a number of paradoxes and contradictions that persisted until the discovery, in the seventeenth century, of calculus. Here some basic concepts of Euclidean geometry are reviewed and the 
several methods of exhaustion practiced by Hippocrates, Euclid and Archimedes are briefly discussed, noting that the latter is closest to the traditions from Mesopotamia, while Euclid remains faithful to the Platonist and Aristotelian tendency.

KeYwords: Exhaustion, Greek Philosophy, Greek Geometry.

\section{Noción de borde o frontera}

La noción de cuerpo limitado y finito, en oposición a lo que es infinito y confuso, porque no tiene límites, condiciona el entramado de la geometría griega: las figuras geométricas necesitan una frontera o borde que las defina. Los límites perfilan y extraen al objeto de su imprecisión, le proporcionan realidad; el espacio se sustancializa cuando se acota. Los conceptos de punto, línea y plano se introducen en la mente como entidades separadas: serán los extremos de la línea, de la superficie y del sólido, respectivamente. El punto es entendido sin magnitud, porque no tiene partes; la línea se extiende en una sola dimensión y el plano lo hace en dos: longitud y anchura.

La geometría elaborada por los matemáticos de las antiguas civilizaciones de Mesopotamia y Egipto, no menciona esos conceptos: no concibe bordes ni extremos de orden inferior. El confín de una superficie es una región de esa misma superficie, no una línea. Y lo mismo sucede con los cuerpos sólidos, los cuales no tienen caras adheridas a modo de cáscara o envoltura. Una línea sería la huella que deja el estilo sobre la tableta de arcilla, la cuerda tendida por el agrimensor para medir una porción de tierra. Un punto solo es una señal, una marca. El plano es como el suelo que pisamos. En la geometría babilónica, las figuras se determinan por sus elementos: el todo se nombra por la parte; idénticas palabras para describir la circunferencia y el círculo (GUR), el rectángulo y su diagonal (siliptum), el cuadrado y su lado (mithartum). La idea de círculo se conceptualiza a partir de la noción de su longitud ${ }^{1}$. Los objetos físicos no adquieren o pierden cualidades al ser representados en un dibujo. No hay abstracciones que desdoblen la realidad en elementos heterogéneos. El escriba de la Antigua Babilonia

\footnotetext{
1 Eleanor Robson (1999).
} 
opera siempre con magnitudes homogéneas; pues, aunque en apariencia sume líneas y superficies, o superficies a sólidos, lo que hace realmente es añadir líneas y planos cuyo espesor es la unidad ${ }^{2}$. Son los indivisibles que más adelante utilizaría Arquímedes.

Concebir un objeto sin extensión y sin partes, como se define el punto en los Elementos, no es sólo una conjetura, sino más bien una hipótesis que se introduce por conveniencia. Porque Platón utilizaba el término hipótesis en el sentido de "condición preliminar de la verdad de lo que se busca» ${ }^{3}$ y Aristóteles afirmaba que las hipótesis garantizan la existencia de los objetos matemáticos simples ${ }^{4}$. También podríamos decir que estas entidades (punto, línea y plano), tal como las entiende Euclides, son únicamente postulados, en el sentido explicitado por Aristóteles ${ }^{5}$, pues dudamos acerca de su existencia y posibilidad, y carecen de significación fuera de la geometría.

\section{El infinito es lo que no tiene límites}

El concepto de infinito no es unívoco. Hallamos un infinito de carácter sustancial, aunque indefinido, en Anaximandro ${ }^{6}$, cuando se refiere a lo ilimitado (ápeiron): aquello que carece de bordes o fronteras y por ello se muestra difuso, sin perfilar; como un paisaje difuminado y perdido en su indeterminación. En Parménides el Ser perfecto y completo abarca todo lo existente; es indivisible e inmutable; definido en sus propios límites. Los pitagóricos observaron el infinito como algo imperfecto y negativo: lo ilimitado; pero sometido al elemento configurador de lo limitado, el cual le da forma y determina, como lo impar limita a lo par; pues todas las cosas en la naturaleza proceden de lo limitado y lo ilimitado.

2 Jens Høyrup (2002).

${ }^{3}$ Menón 86 e.

${ }^{4}$ Aristóteles, Anal. Post. I 2 y en I 10, 76 b: «Lo que es capaz de ser demostrado, pero el maestro lo presupone sin prueba alguna es una hipótesis».

5 Aristóteles, ibid., I 10, 76 b: «el postulado ilegítimo es contrario a la opinión del discípulo", aunque, como la hipótesis, es «una proposición demostrable [que] se supone y se utiliza sin demostración".

${ }^{6}$ Conrado Eggers y Victoria Juliá (1978). 
El denominado por nosotros infinito matemático, lo encontramos en la palabras de Anaxágoras: «En lo pequeño no existe lo mínimo sino sólo algo menor, porque es imposible que el ser se convierta en el No-Ser por división, pero en lo grande existe siempre lo mayor» (fr. 694, p. 343). Haciendo alusión al infinito geométrico, que permite disminuir cada vez más la magnitud, y al aritmético, por el que añadimos un número más al que tenemos.

También, en las multiplicidades de Leucipo y Demócrito y en el Número hay infinito. Ahora, este vocablo no será el sujeto de la oración sino su predicado: Infinito es el número de átomos que invaden el espacio, también inabarcable, según Demócrito; aunque quizá sólo quisiera indicar inconmensurable. Infinita es la cantidad de partes en que podemos fragmentar un cuerpo; infinita es, asimismo, la posibilidad de añadir algo más a lo que ya tenemos. Los filósofos antiguos entremezclaron pensamiento y realidad, lógica y naturaleza, pues jamás alcanzamos cotas infinitas ni en la división ni en la magnitud; tampoco al numerar; ni tenemos constancia de la existencia actual de partículas sin partes o indivisibles. Concebimos cualquier tipo de infinito: la perfección absoluta; la indefinición extrema; la ilimitada divisibilidad de la materia; la inagotable multiplicidad de los átomos y la facultad de numerar sin límite. No obstante, los pensadores antiguos intercambiaron adjetivos y definiciones, tanto del mundo físico como del conceptual, dejando sin concretar cada uno de sus dominios y entendiendo la matemática como mera imagen de lo real: Los átomos del universo se trasladarían al mapa geométrico en forma de puntos, perdiendo en este viaje su magnitud ${ }^{7}$. Los pitagóricos concibieron puntos en el espacio físico y en el matemático, en una cantidad finita. Por el contrario, el continuo postulado por Aristóteles permanecería intacto en la geometría, tan divisible al infinito como su modelo material.

\section{Sobre el pensamiento de Aristóteles}

El filósofo Estagirita definió el infinito en los siguientes términos:

«En un sentido es lo que no puede ser recorrido porque su naturaleza no lo permite, como la voz es invisible; en otro, lo que puede se recorrido de

${ }^{7}$ Elementos I, defs. 1 y 3: «punto es lo que no tiene partes» y «los extremos de la línea son puntos». 
manera incompleta, o que apenas puede serlo, o lo que no puede ser recorrido ni limitado aunque por su naturaleza pueda serlo. Además, todo infinito es o por adición o por división o de ambos modos» ${ }^{8}$.

Admitió únicamente el infinito en tanto proceso o devenir, como potencialidad, es decir, el infinito matemático; rechazando los denominados sustanciales y la posibilidad de actualizar ese infinito potencial ${ }^{9}$ : porque esta acción conlleva la idea de finitud o determinación. Más adelante añade:

«Resulta que lo infinito es lo contrario de lo que se dice. Pues no es aquello fuera de lo cual no hay nada, sino aquello de lo cual siempre hay algo fuera» ${ }^{10}$.

Indicando, además, que «lo continuo se divide al infinito, pero hacia lo mayor no hay infinito» ${ }^{11}$. El continuo de Aristóteles se define como aquello cuyos extremos son una sola cosa y sus partes se diferencian únicamente en cuanto al lugar que ocupan ${ }^{12}$. Puede ser recorrido y también medido, pero nunca habrá una cantidad tan grande que sobrepase los límites de la finitud.

Aristóteles niega la existencia de partículas en el continuo: Los átomos extensos poseen partes: sus bordes, y por tanto, no son indivisibles. Tampoco los puntos inextensos serían los componentes últimos del continuo geométrico, ya que la unión de dos cuerpos se realiza de parte a parte, o de parte a todo, o bien de todo a todo: Los puntos solamente se unirían formando una especie de amasijo, pues al carecer de partes, se añade el todo al todo.

Las tesis indivisibilistas originaron una serie de contradicciones y paradojas; la más llamativa de todas fue la que surgió a raíz de comprobar la inconmensurabilidad entre el lado y la diagonal del cuadrado. Aristóteles, en cambio, recurrió a la dualidad potencia /acto para negar la existencia aqui y ahora de toda clase de infinito; este posee una realidad potencial en el interminable proceso de

\footnotetext{
${ }^{8}$ Física III, 5, 204 a.

9 Del Cielo 5, 7.

${ }^{10}$ Física IV, 6, 206 b-207 a.

${ }^{11}$ Ibid., 207 b.

12 Ibid., VI, 1.
} 
hallar las partes de una extensión. Igualmente, es posible contar indefinidamente partiendo de uno y añadiendo una unidad al número anterior. Jamás obtendremos un número inferior a uno y sí partes del continuo cada vez menores, pues estos son los únicos infinitos potenciales admitidos por Aristóteles: el geométrico en la división y el aritmético en la adición. Descarta, por el contrario, un infinito actual al afirmar que cualquier magnitud se agota si de ella se extrae progresivamente una cantidad constante o alícuota y desmiente a los que defienden el aumento infinito de una extensión, sin tener en cuenta cómo esto supone un infinito exterior del cual se va tomando.

El universo aristotélico es finito, delimitado por la esfera de las estrellas fijas, más allá de la cual no habría nada, ni siquiera el vacío. Su infinito potencial se enmarca en los límites de la magnitud: al sustraer de una cierta cantidad partes progresivamente menores, o cuando añadimos esos mismos términos en cantidad infinita. Aristóteles está aquí aludiendo a las progresiones infinitas, de términos proporcionalmente menores o mayores, respectivamente ${ }^{13}$. Así resuelve las paradojas de Zenón: por muchas partes que atraviese Aquiles, al final recorre una magnitud finita en un tiempo finito. Pues lo contrario: andar una infinidad de partes finitas (por muy pequeñas que éstas sean) supone recorrer una distancia infinita; lo cual es imposible.

\section{Elementos de la geometría euclídea}

La geometría euclídea se encuentra inmersa en un universo platónico. No solo en cuanto a que obedece al formalismo impuesto por la Academia, sino también respecto a los objetos que maneja. Euclides se esmera en crear una obra acorde a la filosofía platónica, sin cómputos ni mediciones, donde sus elementos se muestren eternos, inmutables y ajenos a los vaivenes del mundo sensible ${ }^{14}$. Asignó a los Elementos un orden cronológico que, obviamente, caminaba a la par que sus dificultades intrínsecas. Lo comprobamos en los primeros libros, al no apa-

\footnotetext{
13 De la generación y la corrupción I, 2.

14 "Además de las cosas sensibles y de las Formas, dice [Platón] que hay los objetos de las matemáticas, los cuales ocupan una posición intermedia, diferenciándose de las cosas sensibles por cuanto son eternos e inmutables, y de las Formas por cuanto hay muchos que son semejantes, mientras que la Forma misma es única en cada caso». Aristóteles, Metafisica 987 b.
} 
recer en ellos razones ni proporciones, pero sí la comparación entre unas figuras y otras. Por el contrario, en los libros aritméticos (VII, VIII y IX) se observa una clara influencia mesopotámica y en ellos «no se advierte el menor síntoma de axiomatización» ${ }^{15}$.

\subsection{Magnitud}

En los Elementos no se define el término magnitud, quizá porque a Eudoxo (probable autor de los libros V y VI que tratan de la teoría de la proporcionali$\mathrm{dad})^{16} \mathrm{o}$ al mismo Euclides $^{17}$, no les conviniera incluir un enunciado tan obvio; pero sí se definen razón y número. Comenzaremos expresando la cantidad ${ }^{18}$ como aquello que indica cuánto hay de algo, ya sea discreto o continuo; encontramos cantidad discreta en los agregados de las cosas y cantidad continua en la extensión, el movimiento y el tiempo.

Magnitud es la cantidad extensa, susceptible de medida. Y es magnitud la línea, como también lo son el plano, el sólido y el ángulo, extendidos en las tres direcciones del espacio. La línea es la longitud sin anchura (I, def. 2) y el extremo de una superficie (I, def. 6); posee además una sola dimensión; en cambio, la superficie únicamente tiene dos (I, def. 5) y el sólido tres. El ángulo plano se define como la inclinación de dos líneas (I, def. 8).

15 F. Vera, nota 1 al libro VII de los Elementos, en Científicos griegos, Aguilar, Madrid, 1970.

${ }^{16}$ Se atribuye a Eudoxo la teoría general de la proporción expuesta en los libros V y VI de los Elementos. Aristóteles, de modo indirecto, lo comenta en Anal. Post. I 5, 74 a y II 17, 99 a. También, en los escolios 1 y 3 del libro V, en la edición de Heiberg (1888): Euclidis Opera Omnia V, pp. 280 y 282; en Stamatis (1977), V, pp. 211-213, se comenta que el contenido del libro V se debe a Eudoxo y su disposición a Euclides. En el Catálogo de geómetras (Prologus II, 39), Proclo dice que «Eudoxo de Cnido, un poco más joven que León, se convirtió en miembro de la escuela de Platón y fue el primero en aumentar la cantidad de teoremas llamados "generales", a las tres proporciones conocidas añadió otras tres, acrecentó el número de proposiciones sobre la "sección" —que debe su origen a Platón- e hizo uso del "análisis" para su tratamiento». Proclo (1970), Procli Diadochi in primum Euclidis Elementorum librum commentarii, G. Friedlin (ed.), Leipzig, 1883, reimp. 1967.

Ibid. 67.26: «No mucho más joven [que Hermótimo de Colofón y Filipo de Medma, discípulos de Platón] es Euclides, quien compiló los elementos poniendo en orden varios teoremas de Eudoxo, perfeccionando muchos resultados de Teeteto y dando, asimismo, demostraciones irrefutables de aquello que sus predecesores sólo habían demostrado con escaso rigor».

18 Aristóteles habla de la cantidad en Metafísica X 1, 1053 b. 
Son las tres posibles direcciones de la magnitud las que nos dan una idea del espacio, noción sin definir en los Elementos, pero requisito indispensable de la arquitectura geométrica; realmente, debería figurar como postulado inicial, pero no fue así. El motivo lo hallamos en el dominio que el pensamiento platónico ejerció sobre la concepción de la geometría euclídea: los objetos geométricos no deben situarse en ningún espacio ni en ningún tiempo sensibles; son formas intermedias ubicadas en mundos separados al nuestro.

\subsection{Número}

Cada objeto es una unidad, pues «cada una de las cosas que hay se llama una» ${ }^{19}$. Número (arithmos) es entonces «una pluralidad compuesta de unidades» ${ }^{20}$. Los números, en la geometría de Euclides, son exclusivamente los naturales, y uno no es un número, sino la medida de cualquiera de ellos ${ }^{21}$. Por eso, todo número es parte o partes de otro número ${ }^{22}$. Es parte (méros) cuando lo mide exactamente, como dos es parte de cuatro o de seis; pero ese mismo dos es partes (mere) ${ }^{23}$ de tres y también de cinco, ya que dos son las dos terceras partes de tres, o las dos quintas partes de cinco. Tal operación se puede realizar porque los números poseen una medida común que es la unidad.

En los libros aritméticos (Elementos VII, VIII y IX), los números se representan por líneas. Hay números planos: aquellos que resultan del producto de otros dos; y números sólidos: los que surgen tras multiplicar tres números; cada uno de los números que interviene en el producto se denomina lado ${ }^{24}$. Para Teeteto, quien posiblemente inspiró esta teoría, los números cuadrados [(1·1), (2.2),

19 Elementos VII, def. 1.

${ }^{20}$ Ibid., def. 2.

${ }^{21}$ Aristóteles, Metafísica XIV 3, 1087 b: «La unidad representa la medida de cierta magnitud o pluralidad, y el número es la pluralidad medida y la pluralidad de las medidas, de donde se sigue, con razón, que la unidad no es un número».

${ }^{22}$ Elementos I, def. 3: «Un número es parte de un número, el menor del mayor, cuando mide al mayor» y def. 4: "pero partes cuando no lo mide». (def. 4).

${ }^{23}$ Se refiere a partes alícuotas.

${ }^{24}$ Ibid. VII, defs. 17 a 19. Los pitagóricos distinguieron varias clases de números. Los números cuadrados $(1,4,9, \ldots)$, cuyos respectivos gnomones son $(3,5,7, \ldots)$, es decir, a cada uno de los cuadrados se suma uno de los gnomones, obteniendo el siguiente número cuadrado. Los números triangulares $(1,3,6,10, \ldots)$ y sus gnomones $(2,3,4, \ldots)$. Números pentagonales $(1,5,12,22, \ldots)$ 
(3.3) ...] resultan del producto de igual por igual, y es la figura del cuadrado la que los evoca; mientras los restantes números, se forman del producto de cantidades diferentes $[(1 \cdot 2),(1 \cdot 3),(1 \cdot 5),(2 \cdot 3), \ldots]$, una mayor y otra menor, y los describimos como rectángulos. Así, distinguimos los números cuadrados de aquellos que nombramos oblongos ${ }^{25}$.

\subsection{Medida}

El concepto de medida no se define en los Elementos, aunque lo identificamos con la idea de parte (méros); así, la medida de una magnitud es una parte de esa magnitud ${ }^{26}$ o ella misma. Utilizamos medida y parte, tanto para los números como para las magnitudes. Por el contrario, el concepto de partes (mere) no aparece en relación a la magnitud, pues supondría la posibilidad de que hubiera una medida común a todas las magnitudes ${ }^{27}$, como ocurre con la unidad respecto de los números. Medir es trasladar una magnitud a otra las veces necesarias hasta intentar agotarla ${ }^{28}$. Pero «es conveniente que la medida sea siempre un ser idéntico en especie a los que debe medir ${ }^{29}$; es decir, la longitud se mide con la longitud, la superficie con la superficie y el volumen con el sólido. Si de dos magnitudes desiguales, la menor no es parte de la mayor, se tratará de averiguar si ambas poseen una medida común o, por el contrario, son

y sus gnomones $(4,7,10, \ldots)$. Los números perfectos son aquellos cuya suma de divisores da ese mismo número. Los números amigos son los que, una vez sumados los divisores de uno, obtenemos el otro número. Cf. Morris Kline (1992 I, pp. 53 y ss.).

${ }^{25}$ Teeteto $147 \mathrm{~d}$.

${ }^{26}$ Elementos V, def. 1: «Una magnitud es parte de una magnitud, la menor de la mayor, cuando mide a la mayor». Def. 2: «Y la mayor es múltiplo de la menor cuando es medida por la menor».

${ }^{27}$ Elementos X, def. 1: «Se llaman magnitudes conmensurables aquéllas que se miden con la misma medida, e inconmensurables aquéllas de las que no es posible que haya medida común».

${ }^{28}$ Nos apoyamos en la Noción Común 7 del libro I de los Elementos: "Las cosas que coinciden entre sí son iguales». Este enunciado se conoce como principio de congruencia y justifica la igualdad de las figuras geométricas por el método práctico de epharmózein.

${ }^{29}$ Aristóteles, Metafisica XIV 2, 1087 b y X1, 1053 b.

${ }^{30}$ El descubrimiento de la inconmensurabilidad se halla ligado al intento de los pitagóricos en conseguir la duplicación del cuadrado (Paul Rusnok 1995, p. 114); así consta en Menón 82 bfy Teeteto 147 d. Según Knorr (1998, p. 421): «So that any attempt to describe the first discovery of the incommensurables is necessarily speculative». En Anal. Post. I 23, 41 está la prueba de la inconmensurabilidad de la diagonal del cuadrado. 
inconmensurables una respecto de la otra ${ }^{30}$. Para ello se aplica el procedimiento de antifairesis ${ }^{31}$. Ulteriormente, este método será llamado algoritmo de Euclides. En el libro X, proposición 2 de los Elementos ${ }^{32}$, se define como criterio de inconmensurabilidad:

«Si al restar continua y sucesivamente la menor de la mayor de dos magnitudes desiguales, la restante nunca mide a la anterior, las magnitudes serán inconmensurables».

Aristóteles ya aludió a esta técnica, denominándola antanairesis: «... al demostrar que la línea que corta al plano paralelo a un lado divide, de manera semejante, a la línea que corta y al área, [...] el hecho afirmado resulta inmediatamente evidente, porque se ha restado a las áreas la misma fracción que se había restado a los lados, y ésta es la definición de la misma proporción o semejanza» ${ }^{33}$.

\subsection{Razón y Proporcionalidad}

Medimos o aplicamos antifairesis a las magnitudes homogéneas: «aquellas que al multiplicarse se exceden una a la otra». Pero si dos magnitudes son inconmensurables, podemos establecer una relación —o razón — entre ambas y hacerla después corresponder a otra relación computable y mejor conocida; así Hipócrates de Quíos (h. 470-400) comprobó que los círculos son uno a otro como los cuadrados de sus diámetros respectivos.

Eudoxo expresará juntas la condición de homogeneidad y la idea de razón; lo vemos en el libro $\mathrm{V}$ de los Elementos:

- Definición 3: «Una razón es determinada relación con respecto a su tamaño

${ }^{31}$ Se desconoce el origen de la antifairesis, como técnica de medir. El esquema es el siguiente: $\mathrm{A}=n_{1} m+s_{1} ; m=n_{2} s_{1}+s_{2} ; s_{1}=n_{3} s_{2}+s_{3}$; etc.

32 Fowler (1979, p. 818) afirma que el uso de este algoritmo probó, primeramente, la inconmensurabilidad entre la diagonal y el lado de un pentágono regular (idéntico esquema que el de las magnitudes en extrema y media razón). Creemos que con el método antifairético, Hipasos de Metaponto (s. V a.C.) descubrió la inconmensurabilidad en el dodecaedro (Szabó 1977, p. 32). Knorr (1998, op. cit.), por el contrario, niega que fuera el método antifairético el que desvelara la existencia de magnitudes inconmensurables.

33 Tópicos VIII, 158 b. 
entre dos magnitudes homogéneas».

- Definición 4: «Se dice que guardan razón entre sí las magnitudes que al multiplicarse, pueden exceder una a la otra».

Y restringe el uso de razones a las cantidades extensas y homogéneas, excluyendo no sólo a cualquier otro tipo de cantidades, como puedan ser los números, los movimientos, el tiempo, etc., sino también a las magnitudes heterogéneas entre sí. Hallaremos razón entre pares de líneas, de superficies o de sólidos; pero no entre línea y sólido, superficie y línea ni tampoco entre plano y sólido. Medir, entonces, consistirá en establecer una razón entre dos magnitudes homogéneas para luego buscar su proporción, es decir, para determinar la proporcionalidad existente entre esa razón y una proporción concreta. Por eso decimos que $A$ mide $n$ veces $M$ o que $A$ es a $M$ como $n$ es a 1 : La proporcionalidad se sustenta de razones y proporciones análogas. Cuando los cocientes (o las veces que una magnitud contiene a otra) ${ }^{34}$ de dos esquemas antifairéticos coinciden, sus razones correspondientes son equivalentes $y$, por tanto, las magnitudes respectivas son proporcionales.

\section{Los métodos de exhaución}

Las superficies y los volúmenes de figuras limitadas por curvas contienen un elemento de irracionalidad que dificulta su medida; el deseo de rigor impulsó a los griegos hacia la búsqueda de nuevos procedimientos que facilitasen su cálculo y el más sencillo de ellos consistía en utilizar como instrumento de medida formas rectilíneas conocidas con las cuales establecer una relación de proporcionalidad. Generalmente, procuraban inscribir esa figura curva en un paralelogramo si era plana o, en un prisma, si se trataba de un sólido; pero observaron que aproximando sucesivamente desde su interior polígonos que doblaban el número de sus lados, o paralelepípedos que incrementaban la cantidad de sus caras, lograrían quizás completar esa superficie o ese volumen buscados, consiguiendo así su medi-

${ }^{34}$ Utilizar el vocablo cocientes es anacrónico, porque en la geometría euclídea no hay ninguna operación de división entre magnitudes, sino sustracciones sucesivas; al igual que tampoco hallamos productos entre magnitudes y números, sino repeticiones continuadas de esas mismas magnitudes. 
da. A estas técnicas las denominamos de exhaución, porque pretenden agotar la totalidad de la superficie y el volumen de una figura geométrica curva.

La proposición 1 del libro X de los Elementos fundamenta el método de exhaución ${ }^{35}$ con el que se hallarán cuadraturas y cubaturas:

«Dadas dos magnitudes desiguales, si se quita de la mayor una [magnitud] mayor que su mitad, y de la que queda, una magnitud mayor que su mitad y así sucesivamente, quedará una magnitud que será menor que la magnitud menor dada».

La argumentación de Euclides descansa en la definición 4 del libro V y el resultado, aclara su autor, sería el mismo si tomáramos mitades exactas. Este teorema también es llamado principio de bisección $n^{36}$, porque su técnica reside en duplicar el número de lados de un polígono regular para incrementar su área y restársela después a la superficie cuestionada. Eudoxo fue el primero en enunciarlo ${ }^{37}$ cuando descubrió las proporciones existentes entre las superficies de determinadas figuras y los volúmenes de ciertos sólidos regulares; proporciones que Hipócrates, y también Demócrito, habían advertido anteriormente, pero que no llegaron a demostrar formalmente, según nos cuenta Arquímedes ${ }^{38}$. Euclides incluyó los resultados de Eudoxo en el libro XII. Los teoremas 2, 5, 10, 11, 12 y 18 desarrollan una pauta común.

Antes de Eudoxo, Antifón había intentado hallar la cuadratura del círculo

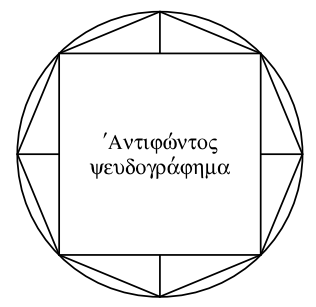

Figura 1

${ }^{35}$ El término exhaución lo sugirió Gregorio de San Vicente, en Theoremata Mathematica (1624), para calificar el método desarrollado por Euclides en el libro XII.

${ }^{36}$ Knorr (1978) distingue el principio de bisección (teorema X.1) del principio de convergencia (X.1 introducido como lema para llevar a cabo el método de exhaución del libro XII y el método de compresión de Arquímedes), y les dio estos nombres. Una diferencia tan sutil que no toda la critica acepta: Dijksterhuis (1987) lo llama principio de dicotomía. Heath (The Thirtenn, op. cit.), Mueller (1981) y Jesseph (1993) no los diferencian. 
inscribiendo en él un cuadrado, luego un octógono, etc., concluyendo que el polígono resultante de infinitos lados debería coincidir con el círculo. Pero únicamente podemos intuir su modo de argumentar, pues nada nos queda de él.

Se atribuye a Hipócrates de Quíos el empleo del método de bisección cuando manifestó que los círculos son uno a otro como los cuadrados de sus diámetros ${ }^{39}$. Hipócrates, según refiere Simplicio ${ }^{40}$, manejaba sumas de infinitos términos, internándose así en el ambiguo terreno del infinito. Theokritos Kouremenos efectúa una reconstrucción de lo que sería su razonamiento:

«Si una magnitud finita puede ser concebida como la suma de 1/2, 1/4, 1/8, $1 / 16$, etc., cada uno de los círculos $k$ y $K$ puede ser también concebido como la suma infinita de $\left(s+\Sigma \tau_{n}\right)$ y $\left(S+\Sigma \Gamma_{n}\right)$, respectivamente; siendo $s$ y $S$ los cuadrados inscritos en $k$ y $K ; \tau_{n}$ y $\Gamma_{n}$, los triángulos obtenidos por bisección en cada uno de los círculos mencionados. Entonces, se cumple que los cuadrados de los diámetros están en la misma razón que los polígonos inscritos en cada uno de los círculos (Elementos XII.1), luego los cuadrados son como esos mismos círculos» ${ }^{41}$.

Hipócrates apoyaría sus tesis en una proposición que después expresaría Eucli-

37 Arquímedes así lo asegura, en: «Carta a Eratóstenes» (El Método), «Carta a Dositeo» (Prefacio Sobre la esfera y el cilindro I) y en el Prefacio de La cuadratura de la parábola.

${ }^{38}$ En La cuadratura de la parábola, Arquímedes afirma que fue Demócrito quien determinó que los volúmenes del cono y de la pirámide son, respectivamente, un tercio de los del cilindro y el prisma de bases iguales e idénticas alturas, pero sin llegar a demostrarlo. Asimismo, Herón, en el prefacio de su Métrica, comenta esto mismo.

39 Eudemo, al comentar que Hipócrates fue el primero en describir la cuadratura de las lúnulas, afirmó que «lo demostró apoyándose en lo que ya había demostrado: que los círculos son entre sí como sus diámetros en potencia», según refiere F. Vera en su edición de los Elementos, Madrid: Aguilar, 1970. Esta proporción figura en Elementos XII, prop. 2.

${ }^{40}$ Simplicio, Simplicii In Aristotelis Physicorum libros quattuor posteriores commentaria consilio et auctoritate, ed. Diels; Academiae Litterarum, Regiae Borussiae. Se basa en la Historia de la Geometría de Eudemo.

${ }^{41}$ T. Kouremenos (1997, p. 232), combina las hipótesis de Knorr y Toeplitz. Éste sostiene que, seguramente, Hipócrates partió de la semejanza entre los polígonos inscritos en una circunferencia y los cuadrados de sus diámetros, para afirmar, entonces, que un círculo debería ser considerado el límite de un polígono de infinitos lados, y establecer así la proporción entre los círculos y sus respectivos cuadrados de los diámetros. Knorr (1982) cree que Hipócrates basó sus razonamientos en una proposición como Elementos V.12. Toeplitz (1925, pp. 175-203).

${ }^{42}$ Es decir: $a: b:: c: d:: e: f:: \ldots:: m: n::(a+c+e \ldots+m):(b+d+f+\ldots+n)$. Aristóteles la menciona en Ética a Nicómaco V 7. 
des en Elementos V.12:
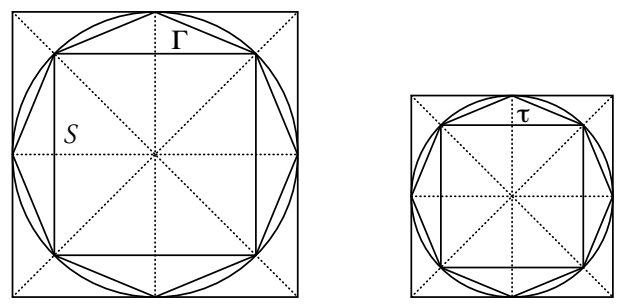

Figura 2

«Si de cualquier número de magnitudes, cada una de las antecedentes tiene la misma razón con cada una de las consecuentes, en la misma razón estarán todos los antecedentes y todos los consecuentes» ${ }^{42}$.

Hipócrates transforma este enunciado en un teorema de sumas infinitas, aunque ya sabemos que el término infinito significa indefinido.

Su razonamiento sería el siguiente:

- Supongamos las siguientes igualdades:

$$
\begin{gathered}
K=S+\Gamma_{1}+\Gamma_{2}+\ldots+\Gamma_{n} \\
k=s+\tau_{1}+\tau_{2}+\ldots+\tau_{n}
\end{gathered}
$$

- Establecemos las razones entre los cuadrados $S$ y $s$ y sus respectivos triángulos $\Gamma_{n}$ y $\tau_{n}$, y aplicamos el enunciado V.12 ${ }^{43}$ :

$$
\begin{aligned}
S: & : s: \Gamma_{1}: \tau_{1}:: \Gamma_{2}: \tau_{2}:: \ldots:: \Gamma_{n}: \tau_{n}::\left(S+\Gamma_{1}+\right. \\
& \left.+\Gamma_{2}+\ldots+\Gamma_{n}\right):\left(s+\tau_{1}+\tau_{2}+\ldots+\tau_{n}\right)
\end{aligned}
$$

— De esta relación de proporcionalidad se infiere que: $S: s:: K: k$

43 Como los polígonos inscritos, a partir del cuadrado, tienen $2^{2}, 2^{3}, 2^{4}$, etc. lados, los $\Gamma$ y $\tau$ son, en realidad: $2^{2}, 2^{3}, 2^{4}, \ldots 2^{\mathrm{n}+1}$ triángulos. Por ejemplo, $\Gamma_{1}$ representa a $2^{2}$ triángulos, $\Gamma_{2}$ a $2^{3}, \ldots$ $\Gamma_{\mathrm{n}}$ a $2^{\mathrm{n}+1}$, etc. 
- Porque

$$
K: k::\left(S+\Gamma_{1}+\Gamma_{2}+\ldots+\Gamma_{n}\right):\left(s+\tau_{1}+\tau_{2}+\ldots+\tau_{n}\right)
$$

O lo que es lo mismo: los círculos son uno a otro como las respectivas sumas de sus figuras inscritas. Sin embargo, ignoramos cómo Hipócrates consumó el resultado previsto, el que afirma que los círculos son como los cuadrados de sus diámetros. Posiblemente fuera esta la causa de que Arquímedes opinara que antes de Eudoxo nadie había logrado demostrar este teorema.

Gracias a esta hipotética reconstrucción reparamos además en otras cosas; por ejemplo, que esos postulados previos [1] indican que un polígono con una cantidad indeterminada de lados, consigue identificarse con el círculo circunscrito. Es decir, que la suma de esos infinitos triángulos, junto al cuadrado inicial, alcan$z a$ el límite que viene señalado por el contorno de la curva. Algo que no se desprende del texto expresado en Elementos X.1, pues aquí la suma indefinida se detiene en cualquier momento y el geómetra se conforma con encontrar una diferencia entre el círculo y el polígono menor que cualquier magnitud dada, fijada de antemano y arbitraria. Por tanto, el modelo concebido por Hipócrates no se ajusta al axioma de congruencia (N.C. 7).

${ }^{44}$ Heath (1956, op. cit., p. 368) muestra el texto recogido por Plutarco (De Comm. Not. adv. Stoicos XXXIX, 3) en el que Demócrito expone la paradoja del cono, según la cual no sabríamos decidir si una lámina, paralela y continua a la base del cono, es o no idéntica a ésta. Heath, apoyado en una frase en la que el filósofo de Abdera afirma que el cono parecerá estar hecho de secciones iguales, asegura que "esto confirma que Demócrito ya tenía la idea de que un sólido está formado por la suma de un infinito número de planos paralelos, o de láminas indefinidamente delgadas, indefinidamente próximas». Y le parece una evidente anticipación de Arquímedes (El Método) y del teorema de Cavalieri (Geometría II, teorema III);sin embargo, el matemático italiano no identifica colecciones de infinitos elementos con sus sumas, ni iguala estas colecciones con las figuras a las que pertenecen; al menos, de un modo explícito.

${ }^{45}$ En los Elementos es el porisma a XII.7: «Una pirámide es 1/3 del prisma que tiene la misma base y altura».

${ }^{46}$ Kouremenos (ibid) compara el razonamiento de Demócrito con el de Newton: «Independientemente de si fue primero avanzado por Demócrito o por Hipócrates, esta técnica guarda una afinidad muy clara con el método de Newton de las primeras y últimas razones de cantidades». El autor alude al corolario del lema IV del libro I de los Principia: «Si dos cantidades de cualquier tipo son divididas de cualquier manera en un número igual de partes, y esas partes — cuando su número es aumentado y su magnitud disminuida hasta lo infinito- guardan una razón dada entre sí, la primera con la primera, la segunda con la segunda y así sucesivamente en orden, todas ellas tomadas conjuntamente guardarán entre sí esa misma razón dada». 
Demócrito determinó el volumen de un cono ${ }^{44}$ de modo parecido a como Hipócrates había razonado respecto del círculo: Tomando el enunciado V.12 y conociendo la relación que guardan entre sí una pirámide y un prisma de la misma base y altura $(1: 3)^{45}$, afirmaría que «como todas las diferencias entre el cono y cada una de las pirámides, y entre el cilindro y cada prisma, respectivamente, están en razón constante, asi el cono y el cilindro están en esa misma razón» ${ }^{46}$.

Difiere, entonces, el principio de bisección aplicado por Hipócrates y Demócrito del lema de exhaución formulado por Eudoxo-Euclides. X.1 soslaya el infinito, no alcanza el límite, únicamente descubre segmentos circulares que la línea poligonal va dibujando en su constante proceso de bisección. Son diferencias tan pequeñas como queramos y, sin embargo, finitas. La propuesta de Eudoxo-Euclides se ajusta, por una parte, al dictum de divisibilidad infinita ${ }^{47}$ y por otra, evita contradecir el principio de homogeneidad expresado en V.4, ya que, por muy numerosos que fueran los lados del polígono inscrito y aumentara su superficie, jamás llegarían a coincidir sus bordes con los de la circunferencia.

En opinión de Kouremenos, ambos geómetras, Hipócrates y Demócrito, sólo precisaban postular que el todo es como la suma de sus partes, pues «no necesitaban hacer explícita una suma infinita; simplemente, deberían haber argumentado que el primer círculo es al segundo como todos los triángulos del primer círculo son a todos los triángulos del segundo círculo ${ }^{48}$, insinuando aqui totalidades semejantes a las propuestas, siglos después, por Cavalieri en su Geometria indivisibilibus $(1635)^{49}$. En realidad, eso fue lo primero que hicieron: identificar el todo con la suma de sus partes y asentar este principio como fundamento de su razonamiento.

Manejar colecciones infinitas significa introducir la noción de un infinito

47 «No existe una magnitud infinita en acto; pero esta magnitud sí podría ser infinita en su divisibilidad», Aristóteles, Fisica III 6, 206.

${ }^{48}$ Kouremenos, ibid., p. 233.

49 Geometria indivisibilibus continuorum nova quadam ratione promota, Bologna: Clemente Ferroni (ed.), 1635. Creemos que Kouremenos alude al corolario del teorema IV del libro II.

${ }^{50}$ «Quienes renunciaron a la división hasta lo infinito por la imposibilidad de dividir las cosas indefinidamente y demostrar que tal división no tiene límite, decían que los cuerpos están formados por partículas indivisibles y se descomponen en éstas", aludiendo a Demócrito y Leucipo. Cf. Diels, Die Fragmente der Vorsokratiker, 1903; Simplicio (54-A-13). 
actual, pero desconocemos si fue esa la verdadera intención de Hipócrates y Demócrito. Tampoco sabemos si al final del proceso de bisección el polígono coincide con el círculo. Los indivisibilistas, pitagóricos y atomistas, creerían que la línea poligonal de infinitos lados acaba resolviéndose en puntos que se superponen a la $\operatorname{curva}^{50}$; por el contrario, la tesis continuista aristotélica defiende que jamás un polígono se identifica con su círculo circunscrito.

El teorema XII.2 de los Elementos es paradigmático respecto al uso del procedimiento de exhaución; con él, Euclides pretende demostrar que «los círculos son uno
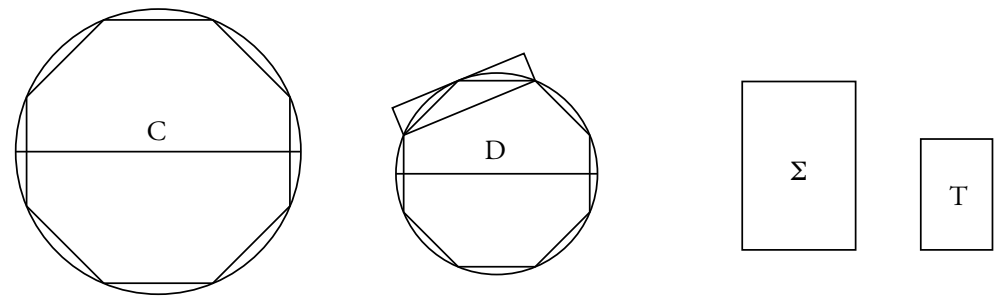

Figura 3: Elementos XII.2

a otro como los cuadrados de sus diámetros». Junto a X.1, incorporado como lema, Euclides introduce un doble razonamiento apagógico de reducción al absurdo. El lema de exhaución impide identificar el círculo con cualesquiera de los polígonos inscritos en él: la diferencia hallada será menor que una magnitud asignada. Precisamente, se distingue del principio de bisección, tal como es manejado por Hipócrates y Demócrito, en la cota que Euclides fija, evitando un proceso ilimitado ${ }^{51}$. El infinito para Euclides es, como para Aristóteles, una posibilidad y no una actualidad ${ }^{52}$.

\section{El axioma de Arquímedes}

${ }^{51}$ Aristóteles manifiesta que «La magnitud se puede dividir siempre por la mitad» (Física III 7, 207 a).

52 El único infinito que concibe Aristóteles es el potencial: «lo continuo se divide al infinito, pero hacia lo mayor no hay infinito» (Física IV 6, 207 b) y «es evidente también que no es posible que el infinito exista como algo dotado de existencia actual» (Fisica III 5, 204 a).

${ }^{53}$ R. Netz (2004). Durán, A. y P. González Urbaneja (2006).

54 Sobre la esfera y el cilindro I, asunción 5. Cf. Dijksterhuis (op. cit., pp 146 y ss.). También, en La cuadratura de la parábola y Sobre las líneas espirales. Luigi Borzacchini (2006). 
Arquímedes ${ }^{53}$ añadió nuevos matices al enunciado X.1. El proceso de inscripción de polígonos a superficies curvas dejaba ciertos residuos sin cubrir que, según su pensamiento, deberían ser homogéneos a los anteriores. Lo expresó así:

«De desiguales líneas, desiguales superficies y desiguales sólidos, el mayor excede al menor por una cantidad tal que, cuando se añade a sí misma, puede exceder cualquier magnitud asignada del tipo de las magnitudes comparadas una con otra ${ }^{54}$.

De esta manera, la línea poligonal nunca alcanzará a la curva ni se identificará con ella; tampoco se resolverá en puntos. Bajo esta idea, subyace otra de carácter filosófico: Arquímedes ignora los bordes de las figuras; éstos no poseen una entidad propia, separada del todo, ni son concebidos con anterioridad a esa totalidad. Las líneas, los planos y los sólidos no contienen elementos heterogéneos de orden inferior. El célebre matemático alejandrino se desvincula de las tesis platónicas ${ }^{55}$ y se sitúa muy cercano a los matemáticos orientales cuando concibe, por ejemplo, las superficies sin aristas y las líneas sin extremos. Al efectuar cuadraturas, utilizará segmentos de anchura unitaria, elementos que ya fueron manejados por sus antecesores babilonios.

El enunciado anterior se conoce como axioma de continuidad o lema de Arquimedes $\mathrm{y}$, al contrario que X.1, fue incluido sin demostración. Su asunción restablece la legitimidad de las construcciones de Hipócrates y Demócrito, garantizando que al introducir el teorema de exhaución no sobrepasaremos el nivel de homogeneidad requerida, aunque para ello hayamos tenido que prescindir de alcanzar el límite que identifica las líneas poligonal y curva ${ }^{56}$.

55 Comenta Proclo: «La mayoría de la gente, observando que esos límites existen imperfectamente en las cosas limitadas, tienen una idea confusa de su ser. Algunos dicen que únicamente son abstraídos de las cosas sensibles por reflexión, otros [creen] que no tienen existencia aparte de nuestros pensamientos. Pero las formas de todos ellos existen en el mundo inteligible, existen en los órdenes del alma, existen en la naturaleza y después de todo, en los cuerpos». (op. cit., 91. 18). Conociendo la afiliación neoplatónica de Proclo, no nos extrañan sus palabras. Arquímedes, por el contrario, no se adhiere a esta opinión.

${ }^{56}$ Dijksterhuis, por el contrario, caracteriza el método de compresión arquimediano como un paso al límite, lo cual contradice el dictum de divisibilidad infinita y el lema X.1 (Ibid., p. 32).

57 Consultar la edición de P. González Urbaneja y Joan Vaqué (1993). 
Arquímedes cuenta en $E l$ Método ${ }^{57}$ a Eratóstenes, cómo consiguió calcular las cuadraturas y cubaturas de algunas superficies y sólidos limitados por líneas curvas:

Pues algunos de los [teoremas] que primero se me hicieron patentes mecánicamente, recibieron luego demostración geométricamente, habida cuenta de que la investigación hecha por este método no comporta demostración; pues es más fácil, después de haber adquirido por ese método cierto conocimiento de la cuestiones objeto de investigación, dar luego la demostración, que investigar sin ningún conocimiento previo... ${ }^{58}$

Es decir, traslada su investigación a una máquina simple, como la balanza, comprobando las relaciones de equilibrio existentes en ella e introduciendo medidas unitarias de peso; éstas, en el contexto de la geometría, son los indivisibles: las líneas y los planos; pero con espesor o anchura, lo cual los hace distintos a los definidos por Euclides.

Expresado de modo sucinto, el método de Arquímedes es el siguiente: primero, establece proporciones geométricas entre segmentos. Después, las transfiere a un hipotético esquema mecánico de carácter estático. A continuación, identifica líneas y pesos, y conjetura que todas las líneas de una determinada figura plana constituyen esa figura y que, asimismo, todos los planos de un sólido com-

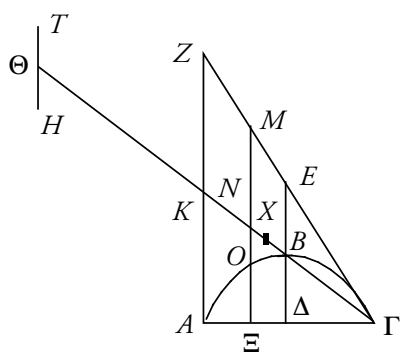

Figura 4

58 «Carta a Eratóstenes», en El Método. El texto fue encontrado por Heiberg en 1906. Aquí observamos claramente la distinción entre justificación y descubrimiento. También, P. González Urbaneja (2008). 
ponen ese sólido. Por último, concluye la cuadratura o cubatura requerida.

Por ejemplo, para demostrar que el área de un segmento parabólico, $S$, es un tercio del triángulo, $\mathrm{AZ} \Gamma$, formado por la recta que abarca la ortótoma, la tangente en uno de sus extremos y la perpendicular en el otro, Arquímedes compone la figura 4, siendo $\mathrm{B} \Delta$ el diámetro y $\mathrm{M} \Xi$ una paralela cualquiera a éste; dibuja $\mathrm{AB}$ y $\mathrm{B} \Gamma$; prolonga ésta última hasta $\Theta$, de tal modo que describa una palanca cuyo centro se sitúe en $\mathrm{K}$, y analiza las razones entre las partes.

La proposición 5 de La cuadratura de la parábola nos da la pauta para expresar la primera proporción:

$$
\mathrm{M} \Xi: \Xi O:: \Gamma A: \Xi A
$$

Por semejanza de triángulos:

$$
\Gamma \mathrm{K}: \mathrm{KN}:: \Gamma \mathrm{A}: \Xi \mathrm{A}
$$

Luego,

$$
\mathrm{M} \Xi: \Xi \mathrm{O}:: \Gamma \mathrm{K}: \mathrm{KN}
$$

$\mathrm{Y}$ como $\Gamma \mathrm{K}=\mathrm{K} \Theta$ :

$$
\mathrm{M} \Xi: \Xi \mathrm{O}: \mathrm{K} \Theta: \mathrm{KN}
$$

Observamos que la expresión [2] también define las relaciones de equilibrio que existirían entre una línea, $\Xi \mathrm{O}$, perteneciente al segmento parabólico y suspendida del extremo de la palanca, $\Theta$, y otra, $M \Xi$, contenida en el triángulo $\mathrm{AZ} \Gamma$ y colocada en el punto $\mathrm{N}$, el cual es el centro de gravedad de la recta $\mathrm{M} \Xi$. $\mathrm{O}$, lo que es lo mismo, hemos hallado la razón entre una línea del segmento y otra del triángulo, resultado que Arquímedes generaliza:

Análogamente, cuantas paralelas a $\mathrm{E} \Delta$ se tracen en el triángulo $\mathrm{AZ} \Gamma$

59 El Método, ed. de L Vega (1986, pp. 41-42). P. González Urbaneja (1993). 
equilibrarán, manteniéndose en su sitio, a los segmentos intersecados sobre ellas por la sección y transportados al punto $\Theta$, de modo que el centro de gravedad de unas y otros sea el punto K. Ahora bien, como las rectas trazadas en el triángulo $\mathrm{AZ} \Gamma$ constituyen el propio triángulo $\mathrm{AZ} \Gamma$ y los segmentos tomados en la sección del mismo modo que $\Xi \mathrm{O}$ componen el segmento $\mathrm{AB} \Gamma$, por ende el triángulo $\mathrm{AZ} \Gamma$, manteniéndose en su sitio, equilibrará respecto del punto $\mathrm{K}$ al segmento de la sección colocado con su centro de gravedad en $\Theta$ de manera que el centro de gravedad de la suma de ambos sea el punto $\mathrm{K}^{59}$.

Una vez establecida la proposición [*], buscamos en $\Theta \Gamma$ un punto $\Xi$, tal que $\Gamma \mathrm{K}=3 \mathrm{~K} \Xi$, con lo cual, $\mathrm{K} \Xi=1 / 3 \Gamma \mathrm{K}$, y por tanto, $\Xi$ es el centro de gravedad del triángulo $\mathrm{AZ} \Gamma$. Podemos formular ahora una nueva relación:

$\Theta \mathrm{K}: \mathrm{K} \Xi:: \nabla \mathrm{AZ} \Gamma: \mathrm{AB} \Gamma$

Es decir, el triángulo $\mathrm{AZ} \Gamma$ se equilibra en su centro de gravedad, $\Xi$, con el segmento parabólico, $\mathrm{AB} \Gamma$, situado en $\Theta$. Y como $\mathrm{K} \Xi=1 / 3 \mathrm{~K} \Gamma=1 / 3 \Theta \mathrm{K}$ :

$\Theta \mathrm{K}: 1 / 3 \Theta \mathrm{K}:: \nabla \mathrm{AZ} \Gamma: S$

Luego

$$
S=1 / 3 \nabla \mathrm{AZ} \Gamma
$$

La clave de este razonamiento reside en el paso inductivo desde una a todas las líneas, y en afirmar que todas ellas conforman el triángulo, en un caso, y el segmento parabólico, en el otro. Arquímedes no se preocupó de la naturaleza de estos indivisibles, ni de su cantidad ni de los problemas epistemológicos que podrían ocasionar en geometría; estas polémicas surgirían siglos después. Tampoco dudó de la equivalencia entre el conjunto de todos ellos y la figura entera; por tanto, aquí sí hay colecciones de un número indeterminado de elementos. Es indiferente si esas colecciones fueron interpretadas como sumas o, simplemente, como su agregado o conjunto, pues argumentando desde la mecánica comprendemos que, efectivamente, la acumulación o suma de cierto número de elementos colocados a un lado

${ }^{60}$ Denominamos infinitésimo a la expresión, ideada por John Wallis en Arithmetica Infinitorum, Opera Mathematica, 1693-1699, vol. I., p. 454. Leer A. Malet (1996, pp. 40-48). También, P. Yuste (2002). 
del fulcro logra equilibrar la carga situada en el otro extremo de la balanza. Arquímedes no menciona expresamente que trabaje con sumas, pues no lo necesita; es suficiente aludir a todos o todas. Pensemos que esos indivisibles arquimedianos tienen su origen en la mecánica y sólo después de experimentar con ellos se incorporan al razonamiento geométrico; toman prestados de esta disciplina sus rasgos y características peculiares, lo cual significa que poseen cierta consistencia física y que no son simples objetos abstraídos de la realidad; en geometría, son entendidos como unidades, y su conjunto, superpuesto a cada figura, conforma un continuo discreto susceptible de descomponerse en esas mismas unidades; de ninguna manera son infinitésimos, aunque su anchura sea mínima o extremadamente pequeña ${ }^{60}$.

La analogía descubierta entre mecánica y geometría permite intercambiar proposiciones entre ambas disciplinas, algo que el formalismo euclídeo nunca hubiera tolerado. Arquímedes fue consciente de esto y de la desconfianza que su método mecánico con indivisibles suscitaba; por tanto, sólo lo utilizó como preámbulo o ensayo de otro más geométrico y formal, como es el de compresión e, incluso, el de aproximación.

Siglos después, Bonaventura Cavalieri, podría haber suscrito las palabras de Arquímedes:

...y todos los triángulos del prisma guardan con todos los triángulos contenidos en el segmento del cilindro la misma razón que todas las rectas del paralelogramo guardan con todas las rectas comprendidas entre la sección de cono rectángulo y la recta... ${ }^{61}$

Aunque Cavalieri no recurrió a la mecánica cuando incorporó indivisibles en sus cuadraturas y sí los concibió al modo euclídeo, es decir, sin anchura. Arquímedes también fue el precursor ignorado del procedimiento utilizado por Evangelista Torricelli en la cuadratura de la parábola; esta vez, inspirado en la mecánica.

${ }^{6}$ El Método, p. 88, donde se determina de modo mecánico la cubatura del segmento de un cilindro. 


\section{Conclusión}

En este trabajo hemos repasado algunos aspectos de la geometría griega, destacando cómo las ideas de punto, línea y plano fueron introducidas, simplemente, como instrumentos de cálculo y para inyectar consistencia a los objetos geométricos. La noción de infinito, en el sentido de lo que es indefinido y sin límites, se excluyó de la geometría: los cuerpos y figuras fueron dotados de extremos y aristas, que los salvaban de la imprecisión. También se rechazó por imposible el infinito actual, que imaginaba la coexistencia de una multitud ilimitada de individuos de la misma clase. Sólo se aceptó el llamado por Aristóteles infinito potencial, que permite la acción de contar y dividir sin término.

La noción de infinito es primordial en la búsqueda de la medida del círculo. La inconmensurabilidad descubierta entre la circunferencia y el diámetro exhorta a los matemáticos griegos a ensayar otros procedimientos de cálculo: si es imposible medir con exactitud, sí al menos, se puede establecer una relación entre magnitudes. Se investiga la razón existente entre la superficie del círculo y el cuadrado de su diámetro. Antifón y Demócrito conciben un nuevo procedimiento: el de exhaución. En dos figuras análogas, aproximan un número indeterminado de polígonos al círculo que los circunscribe, y fijan una relación de proporcionalidad entre sus respectivas superficies. El paso al límite entraña la introducción de sumas infinitas y la identificación de las líneas curva y poligonal, originando inquietantes paradojas. Contrariamente, los principios enunciados por Euclides en los Elementos descartan el uso de infinitos y la posibilidad de igualar el círculo con cualquier polígono inscrito en él, aunque sean muy numerosos sus lados. Así, la dificultad de calcular cuadraturas es evidente y las contradicciones surgidas dejan el tema inmerso en un mar de vaguedad y confusión.

Arquímedes examina esta cuestión desde una perspectiva más realista. Los objetos de la geometría proceden del mundo sensible y conservan sus notas y características; por tanto, carecen de entidades heterogéneas que los limiten. Punto, línea y plano, son elementos que se postulan en geometría para efectuar una construcción eventual y esquemática; indicar una posición en el espacio; señalar una distancia o longitud; determinar un lugar de referencia. Podemos prescindir de su espesor o anchura, si así nos conviene, pero no son los bordes de las figuras geométricas. Posiblemente, el sabio de Siracusa heredó estas ideas de los 
matemáticos orientales, para quienes los objetos físicos poseen siempre tres dimensiones y no hay un infinito que domeñar. El axioma de continuidad incide, precisamente, en este pensamiento; ni siquiera precisa demostración algo tan evidente. El uso de la mecánica supone la transferencia de elementos, proposiciones e hipótesis procedentes del contexto tecnológico hacia el ámbito puramente conceptual y abstracto. La ortodoxia euclídea prohibía estas libertades. Los indivisibles de Arquímedes son como los rectángulos cuya longitud es la unidad y como los sólidos con altitud igual a uno, que manejaban los escribas de Babilonia para legitimar la suma (o resta) de superficie y lado, o de volumen y área. Son coincidencias que reclaman nuestra atención.

\section{Bibliografía}

BorZACCHINI, L. (2006). «Why Rob Archimedes of his Lemma?» Mediterranean Journal of Mathematics 3, pp. 433-448.

CAVALIERI, B. (1635). Geometria indivisibilibus continuorum nova quadam ratione promota, Bologna: Clemente Ferroni, ed.

Dijksterhuis, E. J. (1987). Archimedes, Princeton Univ. Press.

Durán, A. y P. GonZÁlez Urbaneja, eds. (2006): Arquimedes: Obras escogidas, Madrid: RSME.

EGGERS, C.; JuLì̀, V. eds. (1978). Los filósofos presocráticos. Madrid: Gredos.

FowLer, D. H. (1979). "Ratio in Early Greek Mathematics», Bulletin of the AMS 1, n. 6, pp. 807-847.

González Urbaneja, P. y VaQuÉ, J., eds. (1993). Arquímedes. El Método relativo a los teoremas mecánicos, Univ. Autónoma de Barcelona.

González Urbaneja, P. (2008). Arquimedes y los orígenes del cálculo integral, Madrid: Nivola.

Høyrup, J. (2002). Lengths, Widths, Surfaces. A Portrait of Old Babylonian Algebra and Its Kin. New York, Berlin, Heidelberg: Springer-Verlag.

JessePH, D. M. (1993). Berkeley's Philosophy of Mathematics, Chicago Univ. Press.

KuINe, M. (1992). El pensamiento matemático de la Antigüedad a nuestros días. Madrid: Alianza. 
Knorr, W. (1978). "Archimedes and the Pre-Euclidean Proportion Theory», Archives Internationales d'Histoire des Sciences 28, pp. 183-244.

- (1982). «Infinity and Continuity: The Interaction of Mathematics and Philosophy in Antiquity», en Kretzmann, N., ed.: Infinity and Continuity in Ancient and Medieval Thought, pp. 112-145. New York: Ithaca.

- (1998). «Rational Diameters and the Discovery of Incommensurability», American Mathematical Monthly 105, n. ${ }^{\circ}$ 5, pp. 421-430.

Kouremenos, T. (1997). «Mathematical Rigor and the Origin of the Exhaustion Method», Centaurus 39, pp. 230-252.

MaleT, A. (1996). From Indivisibles to Infinitesimals, Barcelona: Enrahonar.

Mueller, I. (1981). Philosophy and Deductive Structure in Euclid's Elements, Cambridge, Mass.

- (1982). "Geometry and Scepticism», en Jonathan Barnes, ed, Science and Speculation, Cambridge: Cambridge Univ. Press.

Netz, R. (2004). The Works of Archimedes, Cambridge. Cambridge Univ. Press.

Robson, E. (1999). Mesopotamian Mathematics, 2100-1600 BC: Technical Constants in Bureaucracy and Education, Oxford: Clarendon Press.

RusNoK, P. (1995). «Strategies for Conceptual Change: Ratio and Proportion in Classical Greek Mathematics», Studies in History and Philosophy of Science 26 / n. . 1, pp. 107-131.

SzABO, A. (1977). Les débuts des mathématiques grecques, París: Vrin.

Toeplitz, O. (1925). «Mathematik und Antike», Die Antike 1.

VeGA, L. ed. (1986). El Método. Madrid: Alianza.

VerA, F. ed. (1970). Científicos griegos, Madrid: Aguilar.

Wallis, John (1693-1699). Arithmetica Infinitorum, Opera Mathematica.

Yuste, P. (2002). «Modelos algebraicos de Wallis para componer sólidos de dimensión infinita y volumen determinado», Éndoxa. Series Filosóficas 16, pp. 333-362.

Recibido: 20/12/2008

Aceptado: 11/01/2009 